Leading Article

\title{
Fluoride treatment in osteoporosis
}

\author{
Pauline Pitt and Hedley Berry
}

King's College Hospital, Denmark Hill, London SE5 9RS, UK

\section{Introduction}

The use of fluoride for the treatment of established vertebral osteoporosis in adults with the symptomatic crush fracture syndrome is approved for use in 8 European countries. ${ }^{1}$ This treatment was suggested by the low prevalence of osteoporosis in some areas where the drinking water contained moderately high concentrations of fluoride ${ }^{2-4}$ and by the enormously increased bone density characteristic of fluorosis. ${ }^{5}$

There now exists a wealth of evidence and some controversy regarding the use of fluoride, and this article addresses the main issues involved.

\section{Pharmacokinetics}

Fluoride is absorbed passively and rapidly from the gastrointestinal tract largely as hydrofluoric acid. ${ }^{6}$ The main site of absorption is the stomach, and in conditions of reduced gastric acidity or concurrent ingestion of milk or calcium this may be reduced. Using sugar-coated sodium fluoride, peak serum concentrations are achieved within $2-3 \mathrm{~h} .{ }^{7}$ Initially $50 \%$ of absorbed fluoride is taken up into bone and the fraction not so utilized rapidly excreted in the urine. $^{8}$

Several preparations of fluoride are available and include both enteric-coated and slow-release preparations in addition to the more simple sugarcoated tablets. Use of the latter twice daily results in 2 peak and trough serum concentrations. The peaks may well exceed toxic concentrations and the troughs be below the therapeutic range. ${ }^{9}$ The use of slow-release preparations may be more useful, giving a peak at around $4 \mathrm{~h}$ and a less rapid decline in serum concentrations, maintaining them within the therapeutic range even $12 \mathrm{~h}$ after ingestion. ${ }^{7}$ There is interesting information evolving about the use of monofluorophosphate and its possible earlier effects on bone density compared with sodium fluoride. ${ }^{10}$

Correspondence: H. Berry, M.A., D.M., F.R.C.P. Received: 9 October 1990

\section{Fluoride in clinical practice}

Fluoride is known to stimulate osteoblasts in culture. ${ }^{11}$ Its use has been associated with increase in alkaline phosphatase activity and it stimulates both collagen synthesis and calcium deposition. ${ }^{11}$ High concentrations may exert a toxic effect on osteoblast function. ${ }^{12}$

At least 4 studies have been carried out to assess the long-term effect of fluoride treatment on spinal bone mass. Some of the results are conflicting. Two prospective controlled trials have been carried out and recently reviewed in detail. ${ }^{13}$ One in France used a daily dose of $50 \mathrm{mg}$ for 2 years and the other in the USA used a high-dose regimen of $60 \mathrm{mg}$ or $90 \mathrm{mg}$ given on alternate days for 4 years.

In the French prospective collaborative study 466 patients ( 421 women and 45 men) between the ages of 50 and 92 years were investigated. ${ }^{14} \mathrm{~A}$ total of 257 received sodium fluoride $50 \mathrm{mg}$ per day ( $25 \mathrm{mg}$ twice daily just before lunch and dinner) for 2 years. The control group of 209 patients was treated with calcium $1 \mathrm{~g} /$ day and vitamin D 800 IU/day (93 patients); calcitonin $50 \mathrm{IU} /$ day for 5 days every 3 weeks and phosphorous $1-1.5 \mathrm{~g} /$ day (85 patients); calcitonin plus calcium (12 patients); calcium plus phosphorous (17 patients); and phosphorous plus etidronate (2 patients). In addition the fluoride group received $1 \mathrm{~g}$ of elemental calcium per day and a vitamin D supplement of $800 \mathrm{IU}$ per day. Of these 466, 316 patients were followed for 24 months. The fluoride group had a significantly reduced crush fracture rate per year compared with controls on other therapies. In addition the mean number of new crush fractures was reduced in the fluoride group but this only reached significance between 12 and 24 months.

There are two prospective double-blind studies recently reported from the USA: one from the Mayo Clinic ${ }^{15}$ and the other from the Henry Ford hospital. ${ }^{16}$ In the Mayo Clinic study 202 women aged between 50 and 75 with postmenopausal osteoporosis were studied. They were required to have one or more vertebral fractures defined as a decrease in vertebral height of greater than $20 \%$. 
As described the treatment group received $60 \mathrm{mg}$ or $90 \mathrm{mg}$ of sodium fluoride on alternate days and the control group was given matching placebo. Both also received $1,500 \mathrm{mg}$ per day of elemental calcium. A total of 135 patients completed the 4 years of therapy. Using dual-photon and absorptiometry and quantitation of the lumbar spine, femoral neck and intertrochanteric region there was a cumulative increase in bone mineral density of $35 \%$ for the lumbar spine, $12 \%$ for the femoral neck and $10 \%$ for the femoral intertrochanteric region. Single photon absorptiometry of the radial shaft showed a reduction of $4 \%$ in the fluoride group. Despite these increases in bone mass at the lumbar spine and femur the $15 \%$ reduction in the number of women experiencing a new crush fracture in the fluoride group was not statistically significant. There was also no relationship between the changes in bone mass of the lumbar spine and the fracture rate.

The Henry Ford Hospital study, again using $60 \mathrm{mg}$ or $90 \mathrm{mg}$ of fluoride on alternate days with calcium supplementation, investigated 84 osteoporotic females with at least one radiographic vertebral fracture. There was no quantitation of bone density and a new vertebral fracture was defined as in the Mayo Clinic study with bone scans being used to detect new fractures. Seventy-five patients completed a 4-year treatment period; 529 new vertebral fractures per 1,000 patient years were observed in the non-fluoride group and 718 in the fluoride group. The rates were not significantly different.

The American studies do not suggest that the use of high-dose fluoride therapy is associated with a reduction in the incidence of vertebral fracture despite the evidence for increased bone density in the Henry Ford study. The use of lower-dose treatment with calcium and vitamin D supplementation as described by the French collaborative study would support this use of fluoride in reducing the incidence of new vertebral fractures when compared with other forms of therapy as described.

\section{Risks}

There is some concern that the quality and hence mechanical characteristics of the new bone laid down may be abnormal, even though, as discussed above, it is frequently associated with increased bone density. Certainly severely fluorotic bone is stronger in compression ${ }^{17}$ but weaker in tension than normal bone. ${ }^{18}$ It appears that the effect of fluoride on bone depends on the dose used and whether it is given with calcium. Histomorphometric analysis has shown that high-dose therapy, especially without calcium supplementation, can be associated with osteomalacia ${ }^{19}$ and abnormal fibrous or mosaic bone may occur. This being the case it is not recommended that high doses be used. However, in situations where low-dose fluoride is given with calcium supplementation in vitamin D-replete subjects, histomorphometry shows the newly formed bone matrix to be of normal appearance and adequately mineralized. ${ }^{20}$

Some authors have suggested that there may be an increased incidence of fractured neck of femur in patients on fluoride therapy. ${ }^{15}$ Interestingly these patients had received high doses (average daily dose of $75 \mathrm{mg}$ ) of fluoride, and as yet the authors have not published the results of the bone histomorphometry in these subjects. Other investigators $^{14}$ using lower doses with calcium and vitamin D supplementation have not found any such problems.

\section{Side effects}

Gastroenterological side effects are common, occurring in between 10 and $40 \%$ of patients, and appear to be both dose and preparation dependent. ${ }^{7}$ The most frequent complaints are nausea and vomiting, although mild gastrointestinal bleeding may occur. ${ }^{1}$ With the use of sugar-coated sodium fluoride, gastroscopy of patients revealed a high incidence of abnormality although no control subjects were examined. ${ }^{21}$ In low dosage, i.e. less than $50 \mathrm{mg}$ per day, and with enteric-coated tablets, there was no difference between patients and controls. ${ }^{14}$ In individual patients symptoms can generally be relieved by giving the fluoride after meals, lowering the dose or changing to entericcoated or slow-release preparations.

Transient lower extremity pain syndrome occurs in up to $50 \%$ of patients, ${ }^{22}$ although some investigators have found similar incidences of episodic osteoarticular pain in osteoporotic patients being treated with and without fluoride..$^{14}$ Nonetheless, the incidence of pain in the ankle and foot was higher in those on fluoride and may be due to fatigue fractures, which can progress to clinical breaks. Radionuclear bone scan may be a useful investigation, showing increased uptake in the calcium of patients in whom X-ray is normal. ${ }^{23}$ The management is temporary discontinuation of fluoride therapy but maintenance of calcium supplementation. Interestingly, in one study patients who developed this complication had greater increases in lumbar spine bone mineral density than those who did not. ${ }^{10}$

\section{Monitoring therapy and the problem of non-responders}

Approximately $20 \%$ of patients do not appear to respond to fluoride therapy with a change in bone 
mass. ${ }^{1}$ Some authors suggest that this may be a reflection of poor absorption from the gut and hence lower serum fluoride levels and reduced skeletal fluoride content. ${ }^{24}$ Previously patients have been monitored by measurement of changes in alkaline phosphate from baseline in response to treatment, ${ }^{25}$ although not all investigators have found this helpful. An interesting and more sensitive index may be serum osteocalcin measurement. ${ }^{26}$ Certainly markedly increased concentrations have been associated with fluorosis and more modest elevations may predict response.

In terms of preventing side effects as well as predicting response to therapy, the use of serum fluoride concentrations can be helpful. ${ }^{27}$ It appears that there is a narrow therapeutic window, and serum fluoride concentrations need to be at least $95 \mathrm{ng} / \mathrm{ml}$ before a beneficial effect on bone mass can be obtained and below $190 \mathrm{ng} / \mathrm{ml}$ to avoid rheumatological complications. ${ }^{28}$ The pattern of absorption varies depending on the preparations used as discussed above and this needs to be considered in such monitoring.

\section{Conclusions}

Despite recent reports concentrating on the problems associated with high-dose fluoride regimens, ${ }^{29}$

\section{References}

1. Heaney, R.P., Baylink, D.J., Johnston, C. et al. Fluoride therapy for the vertebral crush fracture syndrome. A status report. Ann Intern Med 1989, 111: 678-680.

2. Leone, N.C., Stevenson, C.A., Hilbish, T.F. \& Sosman, M.C. A roentgenologic study of a human population exposed to high-fluoride domestic water. Am J Roentgenol 1955, 74: 874-885.

3. Bernstein, D.S., Sadowsky, N., Hegsted, D.M. et al. Prevalence of osteoporosis in high- and low-fluoride areas in North Dakota. JAMA 1966, 198: 85-90.

4. Simonen, O. \& Laitinen, O. Does fluoridation of drinking water prevent bone fragility and osteoporosis? Lancet 1985 , ii: $432-434$.

5. Singh, A., Jolly, S.S., Bansal, B.C. \& Mathur, C.C. Endemic fluorosis: epidemiological, clinical and biochemical study of chronic fluorine intoxication in Panjab (India). Medicine 1963, 42: 229-246.

6. Whitford, G.M. \& Pashley, D.H. Fluoride absorption: the influence of gastric acidity. Calcif Tissue Int 1984, 36: 302-307.

7. Pak, C.Y.C., Sakahaee, K., Gallagher, C. et al. Attainment of therapeutic fluoride levels in serum without major side effects using a slow release preparation of sodium fluoride in postmenopausal osteoporosis. J Bone Min Res 1986, 1: $563-571$.

8. Hodge, H.C. \& Smith, F.A. Fluoride. In: Bronner, F. \& Coburn, J.W. (eds) Disorders of Mineral Metabolism, Vol 1. Academic Press, New York, 1981, pp. 439-481.

9. Ekstrand, J., Alvan, G., Borens, L.V. \& Norlin, A. Pharmacokinetics of fluoride in man after single and multiple oral doses. Eur Clin Pharmacol 1977, 12: 311-317. fluoride therapy for established vertebral crush fracture syndrome has been shown to be of value and associated with an increase in bone density and a reduced incidence of vertebral fracture. Patients should be monitored carefully, preferably using fluoride concentrations and a measurement of osteoblastic response such as osteocalcin or the more recently developed quantitation of type 1 collagen synthesis available from measurement of procollagen peptide. ${ }^{30}$ Only low doses are recommended for use in patients who are vitamin D replete and receiving calcium supplementation.

Recently interest in the intermittent use of the bisphosphonate, etidronate, has been stimulated by several studies showing its efficacy in preventing bone loss in postmenopausal women. ${ }^{31,32}$ It is an inhibitor of bone resorption and in these patients small changes were noted in bone density. Fluoride therapy, however, remains the only known anabolic treatment for osteoporosis apart from anabolic steroids with their well-documented side effects of liver toxicity. ${ }^{33}$ Despite concern from a few centres about a possible increased incidence of hip fracture in patients on high-dose treatment, this form of therapy should not be abandoned.

0. Delmas, P.D., Dupuis, J., Duboeuf, F. et al. Treatment of vertebral osteoporosis with disodium monofluorophosphate: comparison with sodium fluoride. J Bone Min Res 1989, 5 (Suppl 1): S143-147.

11. Farley, J.R., Wergedal, J.E. \& Baylink, D.J. Fluoride directly stimulates proliferation and alkaline phosphatase activity of bone forming cells. Science 1983, 222: 330-332.

12. Kanis, J.A. \& Meunier, P.J. Should we use fluoride to treat osteoporosis? A review. $Q J$ Med 1983, 210: 145-164.

13. Meunier, P.J. Fluoride and osteoporosis. Bone 1990, 7: 63-66.

14. Mamelle, N., Meunier, P.J., Dusan, R. et al. Risk-benefit ratio of sodium fluoride treatment in primary vertebral osteoporosis. Lancet 1988, ii: $361-365$.

15. Riggs, B.L., Hodgson, S.F., O'Fallon, W.M. et al. Effect of fluoride treatment on the fracture rate in post-menopausal osteoporosis. N Engl J Med 1990, 322: 802-809.

16. Kleerekoper, M., Peterson, E., Phillips, E. et al. Continuous sodium fluoride therapy does not reduce vertebral fracture rate in postmenopausal osteoporosis. J Bone Min Res 1989, 4 (Suppl): S376.

17. Beary, D.F. The effects of fluoride and low calcium on the physical properties of the rat femur. Anat Rec 1969, 164: 305-316.

18. Riggins, R.S., Zeman, F. \& Moon, D. The effects of sodium fluoride on bone breaking strength. Calcif Tissue Res 1974, 14: $283-289$. 
19. Meunier, P.J., Galus, K., Briancon, D. et al. Treatment of primary osteoporosis with drugs that increase bone formation: sodium fluoride, hPTH 1-43, ADFR concept. In: Christiansen, C., Arnaud, C.D., Nordin, B.E.C., Parfitt, A.M., Peck, W.A. \& Riggs, B.L. (eds) Osteoporosis, (International Symposium on Osteoporosis). Department of Clinical Chemistry Oylosbrun Hospital, Copenhagen, Denmark, 1984, pp. 595-602.

20. Zerwekh, J.E., Sakhae, K., Johnson, K.D. \& Pak, C.Y.C. Histomorphometric evaluation of intermittent fluoride therapy in post-menopausal osteoporosis. 34th Annual Meeting, Orthopaedic Research Society. Atlanta, Georgia, 1988.

21. Spak, C.-J., Sjostedt, S., Eleborg, L. et al. Tissue response of gastric mucosa after ingestion of fluoride. $\mathrm{Br} \mathrm{Med} J$ 1989, 298: 1686- 1687.

22. Dambacher, M.A., Huer, J. \& Ruegsegger, P. Long-term therapy of postmenopausal osteoporosis. Bone 1986, 7: 199-205.

23. O'Duffy, J.D., Wahner, H.W., O'Fallon, W.M. et al. Mechanism of acute lower extremity pain syndrome in fluoride-treated osteoporotic patients. Am J Med 1986, 80: $561-566$.

24. Budden, F.H., Bayley, J.E., Harrison, J.E. et al. The effect of fluoride on bone histology in postmenopausal osteoporosis depends on adequate fluoride absorption and retention. $J$ Bone Min Res 1988, 3: 127-132.
25. Farley, S.M.G., Wergedal, J.E., Smith, L.C. et al. Fluoride therapy for osteoporosis: characterization of the skeletal response by serial measurements of serum alkaline phosphatase activity. Metabolism 1987, 36: 211-218.

26. Dandonna, P., Gill, D.S. \& Khokher, M.A. Fluoride and osteoblasts. Lancet 1989, i: 449-450.

27. van Kesteren, R.G., Duursma, S.A., Visser, W.J. et al. Fluoride in serum and bone during treatment of osteoporosis with sodium fluoride and vitamin D. Metab Bone Dis Rel Res 1982, 4: 31-37.

28. Taves, D.R. New approach to the treatment of bone disease with fluoride. Fed Proc 1982, 29: 1185-1187.

29. Smith, R. Osteoporosis after 60. Br Med J 1990, 301: $452-453$.

30. Melkko, J., Niemi, S., Risteli, L. \& Risteli, J. Radioimmunoassay of the carboxy terminal propeptide of human type 1 procollagen. Clin Chem 1990, 36: 1328-1332.

31. Storm, T., Thamsborg, G., Steiniche, T., Genant, H.K. \& Sorensen, O.H. Effect of intermittent cyclical etidronate therapy on bone mass and fracture rate in women with post-menopausal osteoporosis. N Engl J Med 1990, 322: $1265-1271$.

32. Watts, N.B., Harris, S.T., Genant, H.K. et al.,. Intermittent cyclical etidronate treatment of post-menopausal osteoporosis. $N$ Engl J Med 1990, 323: 73-79.

33. Consensus development conference: prophylaxis and treatment of osteoporosis. Br Med J 1987, 295: 914-915. 